\title{
The (head and neck) carcinologic handicap index: validation of a modular type questionnaire and its ability to prioritise patients' needs
}

\author{
Mathieu Balaguer ${ }^{(1,2)}$, Maëlle Champenois ${ }^{(3)}$, Jérôme Farinas ${ }^{(2)}$, Julien Pinquier ${ }^{(2)}$, Virginie Woisard \\ $(1,4)$ \\ (1) CHU Larrey, Toulouse, France \\ (2) IRIT, CNRS, Université Paul Sabatier Toulouse III, Toulouse, France \\ (3) Faculté de médecine Toulouse Rangueil, Université Paul Sabatier Toulouse III, Toulouse, \\ France \\ (4) Laboratoire Octogone-Lordat, Toulouse, France
}

\section{Contact information of the corresponding author:}

\section{Mathieu Balaguer}

IRIT Institut de Recherche en Informatique de Toulouse

118 Route de Narbonne

31062 TOULOUSE CEDEX 9

mathieu.balaguer@irit.fr

$+33688163284$

ORCID : 0000-0003-1311-4501

\begin{abstract}
$\underline{\text { Abstract }}$
Purpose: To validate the upgraded version of the CHI with two new dimensions ("limitation of neck and/or shoulder movements", "changes in physical appearance"). To assess the relationship between CHI scores and patient self-reported management needs.

Methods: 71 patients treated for cancer with ENT complaints and 36 controls were included. Construct validity, internal consistency, criterion validity (using visual analogue scales by dimension), clinical validity (comparison of patient and control scores) and temporal reliability (scores of a second CHI completed after a few days) were studied. A hierarchical ranking of the dimensions according to perceived difficulties was compared to the CHI scores.

Results: Correlations were moderate to high between items of the same dimension $(0.38<\mathrm{r}<0.73)$, between scores on the two new dimensions and on the VAS ( $r>0.68)$, and between scores on the two CHI completions ( $r>0.67)$. Cronbach's alphas are greater than 0.72. Patients and controls had significantly different scores in the two new dimensions (Mann-Whitney: p<0.001). Sensitivity, specificity and AUC calculated between CHI scores and hierarchy ranking determined patient priority cut-off scores for eight of the eleven dimensions.

Conclusion: The new CHI dimensions have good psychometric qualities. Threshold scores by dimension allow the perceived management needs to be determined.
\end{abstract}

\section{$\underline{\text { Keywords }}$}

aerodigestive tract cancer; quality of life; handicap; self-assessment 


\section{$\underline{\text { Declarations }}$}

Funding: This study was funded by the Hospitals of Toulouse, and Grant ANR-18-CE45-0008 from The French National Research Agency in 2018 RUGBI project "Improving the measurement of intelligibility of pathological production disorders impaired speech" lead by Jérôme Farinas at IRIT (Institut de Recherche en Informatique de Toulouse).

Conflicts of Interest: All authors declare that they have no conflict of interest.

Ethics approval: All procedures performed in studies involving human participants were in accordance with the ethical standards of the institutional and national research committee and with the 1964 Helsinki declaration and its later amendments or comparable ethical standards.

Consent to participate: Each subject was told in advance of the purpose of this study and was given an information sheet. The subjects' non-opposition was obtained.

Consent for publication: Not applicable

Availability of data and material: The database is available on request to the corresponding author.

Code availability: Not applicable

Authors' contributions: Virginie Woisard and Mathieu Balaguer conceived of the presented idea. Maëlle Champenois carried out the experiment. Mathieu Balaguer and Virginie Woisard helped supervise the project (conceive and plan the experiments). Mathieu Balaguer, Maëlle Champenois and Virginie Woisard contributed to the interpretation of the results. Mathieu Balaguer and Maëlle Champenois wrote the manuscript with the support of Jérôme Farinas and Julien Pinquier. All authors provided critical feedback and helped shape the research, analysis and manuscript. 
Purpose: To validate the upgraded version of the CHI with two new dimensions ("limitation of neck and/or shoulder movements", "changes in physical appearance"). To assess the relationship between $\mathrm{CHI}$ scores and patient self-reported management needs.

Methods: 71 patients treated for cancer with ENT complaints and 36 controls were included. Construct validity, internal consistency, criterion validity (using visual analogue scales by dimension), clinical validity (comparison of patient and control scores) and temporal reliability (scores of a second CHI completed after a few days) were studied. A hierarchical ranking of the dimensions according to perceived difficulties was compared to the $\mathrm{CHI}$ scores.

Results: Correlations were moderate to high between items of the same dimension $(0.38<r<0.73)$, between scores on the two new dimensions and on the VAS ( $r>0.68)$, and between scores on the two CHI completions ( $r>0.67)$. Cronbach's alphas are greater than 0.72 . Patients and controls had significantly different scores in the two new dimensions (MannWhitney: $p<0.001$ ). Sensitivity, specificity and AUC calculated between CHI scores and hierarchy ranking determined patient priority cut-off scores for eight of the eleven dimensions.

Conclusion: The new CHI dimensions have good psychometric qualities. Threshold scores by dimension allow the perceived management needs to be determined.

$\underline{\text { Main article }}$

\section{Introduction}

Upper aerodigestive tract (UAT) cancers are common forms of cancer. The mortality rate associated with this type of cancer is decreasing thanks to advances in medical research. More and more individuals are living with the treatment-related sequelae of these conditions or are developing late-onset side effects. Quality of life is negatively impacted $[1,2]$ by altering key functions in the ENT domain.

Furthermore, symptom evaluation does not always predict the impact a given symptom will have on how an individual functions. The impact of treatment sequelae on daily life differs from one person to the next $[3,4,5]$. Nowadays, consideration of quality of life measures in clinical practice appears to be of paramount importance in order to individualise, optimise and coordinate the management strategy offered to patients. For example, the priority of some laryngectomized patients is to be able to eat a normal diet, when swallowing abilities are degraded after radiotherapy, more than getting a new voice. Similarly, the identification of deficits related to neck or shoulder restrictions will allow earlier and more effective referral to a physiotherapist.

Numerous studies have highlighted the benefits to practitioners when these measures are readily available [6].

Many tools have been developed for this purpose, particularly in oncology: the European Organisation for Research and Treatment of Cancer (EORTC) Quality of Life Questionnaire or University of Washington Quality of Life (UWQOL) Questionnaire [7]. Additional modules, specific to tumour sites, such as the Head and Neck 35 relating to cancers [8], are also available. Other handicap indices specific to a particular function are also used in clinical practice, such as the Speech Handicap Index (SHI) [9] or the Phonation Handicap Index [10] which address the functional impact on speech. The concept of the "Patient Concerns Inventory" (PCI) [11] has also been developed at the same time. This questionnaire is used to 
identify the concerns that patients want to raise during their follow-up appointment. The benefits of such tools have been demonstrated in current clinical practice [12]. The Carcinologic Handicap Index was developed along these lines [13]. It is aimed at assessing the perceived symptoms in the UAT area after cancer treatment, regardless of the initial location of the tumour. To that end, the $\mathrm{CHI}$ can be considered as a generic tool in cancerology. The studies carried out with the first version of the CHI showed that the questionnaire has good psychometric features which corroborate its use in clinical practice [13]. However, the practical implementation of this tool highlighted the absence of two dimensions that often pose problems for patients: limitation of neck and/or shoulder movements and the psychosocial impact of changes in physical appearance. To address this, the $\mathrm{CHI}$ has been upgraded to include these two new domains. In addition, the PCI approach now begs the question whether the CHI can be used to determine patient priorities in implementing appropriate management strategies.

Thus the main purpose of this study was to validate the upgraded version of the Carcinologic Handicap Index following the addition of the two new dimensions. A second objective for ecological purposes focused on highlighting the link between CHI scores and patients' needs in terms of management strategy.

\section{Material and method}

\subsection{Inclusion and non-inclusion criteria}

This observational, prospective, cross-sectional questionnaire validation study was carried out between November 2017 and March 2018.

Current cancer patients or those who previously had cancer (ADT cancer or another site) attending the Oncology Rehabilitation Unit at the Toulouse University Cancer Institute and presenting an ENT problem to justify the consultation, were enrolled in the study.

No restrictions were imposed in terms of the tumour site, stage or characteristics of the disease, or the type of treatment administered.

A "control" population was also recruited at the same time. This control tended to be matched to the patient group as closely as possible in terms of age, gender and socio-professional category in order to compare the answers of these diverse groups to the questionnaire. So, this control cohort was recruited from individuals accompanying patients and from the families of speech therapy students.

Each patient was told in advance of the purpose of this study and was given an information sheet. The subjects' informed consent was obtained.

\subsection{Data collected}

The subjects (patients and controls) completed an initial CHI on enrolment. The upgraded version of the $\mathrm{CHI}$ was distributed: in addition to the nine initial dimensions (pain, swallowing, feeding, respiration, phonation, hearing, vision, olfaction-gustation, psychosocial), it also included the two new dimensions, "limitation of neck and/or shoulder movements" and "changes in physical appearance" (see Appendix 1).

These two new domains were created along the same lines (validity of content) as the original design: an initial drafting phase in discussions with the nursing team to generate relevant questions to ask in each new domain. A second patient testing phase was carried out to check the relevance and assess the clarity and comprehensibility of the questions. 
Each of the eleven dimensions included four questions modelled on the Likert 5-point scale. For each question, the subjects had to tick a box that best described the difficulty they perceived (ranging from 0 "never" to 4 "always"). This generated a score for each item (from 0 to 4 ) and a sub-score per domain corresponding to the sum of the scores obtained in relation to the questions asked in that domain (from 0 to 16). The higher the score obtained, the greater the difficulty perceived by the patient.

The scores given by the patients and controls in this first $\mathrm{CHI}$ were compared to check clinical validity. The patient scores were used to study construct validity by analysing the score correlation matrix for inter- and intra-dimension items, and a confirmatory factor analysis conducted a posteriori.

The patients also completed a visual analogue scale (VAS) for each dimension. Criterion validity was investigated by comparing the VAS and CHI scores for each dimension.

The patients were given a second CHI and stamped envelope on enrolment in the study. The patients were asked to complete this second CHI seven to fifteen days later, which allowed temporal stability (test-retest reliability) to be studied.

Finally, the patients ranked the $11 \mathrm{CHI}$ dimensions, as instructed below, on the day of enrolment in order to study the link between the CHI results and the patients' needs: "Please rank the following symptoms in order, from most problematic to least problematic, depending on your own feelings at this time". A ranking was then established, with a value of 1 being allocated to the priority dimension (the one placed by the patient at the top of the list) and 11 to the dimension considered by the patient to be least important. To compare this ranking against $\mathrm{CHI}$ scores, the $\mathrm{CHI}$ dimensions were also ranked. A value of 1 was allocated to the dimension with the highest sub-score and 11 to the lowest sub-score. The performance of the $\mathrm{CHI}$ in terms of screening priority patient management dimensions was then investigated. Any score greater than or equal to a dimension cut-off value is the score from which the dimension can be considered a priority for the patient.

\subsection{Data analysis}

Non-parametric statistical tests were used given the non-Gaussian distribution of the data (assessed using Shapiro-Wilk tests).

The terminology used complies with COSMIN recommendations [14].

Construct validity (study of score correlations per inter- and intra-domain item, criterion validity (sub-scores and VAS) and temporal stability were thus assessed using Spearman's rank correlation coefficients.

Clinical validity in terms of the difference between patient and control scores was investigated using Mann-Whitney's U test. The level of significance was established at 0.005 . The results with $p$ values ranging from $0.005 \leq p \leq 0.05$ were deemed to be suggestive whereas results with a $\mathrm{p}$ value greater than or equal to 0.05 were considered insignificant $[15$, $16]$. Internal consistency was measured using Cronbach's alpha coefficients.

Regarding the secondary objective, a statistical comparison of the scores obtained in the questionnaire and the subjective ranking of the dimensions based on the difficulties perceived by the patients themselves was carried out by analysing intraclass correlation coefficients. The CHI outcome in terms of screening priority patient domains was determined by means of an ROC (Receiver Operating Characteristic) curve analysis to facilitate the practical application of this questionnaire. To this end, a binary variable was created. A value of 1 was allocated if the domain ranked in the top three highest scores on completion of the patient 
questionnaire, and 0 if it ranked between positions four and eleven. Thus, the area under the curve could be calculated for each domain (with its 95\% confidence interval) alongside performance in terms of sensitivity, specificity and proportion of items ranked correctly for domain can be deemed a management priority by the patient: sensitivity and specificity had to be greater than $50 \%$ with at least $50 \%$ of items ranked correctly.

Stata 14.2 software was used for all statistical analyses.

\section{Results}

\subsection{Population}

Seventy one (71) patients (46 men and 25 women with an average age of 64.5) were enrolled in the study (Table 1).

The length of time since completion of treatment ranged from zero months (currently on follow-up) to 240 months (20 years). The length of time since completion of treatment ranged from zero to 21 months for 50 percent of patients.

$19.7 \%$ of patients were treated for a tumour in the oral cavity, $21.1 \%$ for the larynx, $29.6 \%$ for a pharyngeal tumour and $7 \%$ for an ENT tumour in a different location (thyroid or cervical adenopathy with no primary site). $22.6 \%$ of patients had been treated for cancer in another location but presented an ENT problem leading to the consultation.

Thirty-six (36) controls (19 women and 17 men with an average age of 59.5) were also enrolled in the study (Table 1).

\subsection{Construct validity: convergent and discriminant validity for the two new dimensions}

In terms of convergent validity, the inter-item correlation coefficients in the same domain ranged from 0.35 to 0.61 for "limitation of neck and/or shoulder movements" and from 0.37 to 0.74 for "changes in physical appearance".

With regard to discriminant validity, for the "limitation of neck and/or shoulder movements" dimension, correlation scores below 0.35 (i.e. the lowest intra-domain correlation for this domain) were recorded for 156 items out of 160 (97.5\%). For the "Changes in physical appearance" dimension, correlation scores below 0.374 (lowest intra-domain correlation for this domain) were recorded for 136 items out of 160 (85\%) (Table 2).

Confirmatory factor analysis revealed strong correlations between the items in each dimension for seven out of eleven dimensions whereas the "Changes in physical appearance" and "Psychosocial" items were strongly correlated and regrouped in the same factor. The same applies to the items in the "Swallowing" and "Feeding" dimensions.

\subsection{Clinical validity: comparison of patient and control scores}

A significant difference in scores $(\mathrm{p}<0.001)$ was recorded between patients and controls for eight out of the eleven Carcinologic Handicap Index dimensions (Swallowing, Feeding, Phonation, Respiration, Olfaction/Gustation, Psychosocial, Limitation of neck and/or shoulder movements, Changes in physical appearance). A suggestive difference between patients and controls was noted with regard to the Hearing dimension. There was no significant difference between the Pain and Vision dimensions. With specific focus on the two new dimensions, a mean score of 7.23 (standard deviation: 4.75) was recorded for patients and a mean score of 1.61 (standard deviation: 2.59 ) for the 
controls (Mann-Whitney, $\mathrm{p}<0.001)$ for the "Limitation of neck and/or shoulder movements" dimension compared to mean scores of 4.87 (standard deviation: 4.45) for patients and 0.55 (standard deviation: 0.91) for the controls (Mann-Whitney, $\mathrm{p}<0.001$ ) for the "Changes in physical appearance" dimension.

\subsection{Criterion validity}

Spearman's correlation coefficients between the score obtained in the CHI domain and the VAS score for the corresponding domain were calculated.

A strong correlation between $\mathrm{CHI}$ and VAS was recorded for both new dimensions $(\mathrm{r}=0.74$, $\mathrm{p}<0.001$ for "Changes in physical appearance" and $\mathrm{r}=0.68, \mathrm{p}<0.001$ for "Limitation of neck and/or shoulder movements").

\subsection{Reliability of the questionnaire: internal consistency and temporal stability}

High Cronbach alphas were recorded for the two new dimensions. Alphas ranging from 0.75 to 0.83 and from 0.73 to 0.79 were documented for the "Changes in physical appearance" and "Limitation of neck and/or shoulder movements" dimensions, respectively.

With regard to temporal stability, 58\% of the questionnaires were returned (41 questionnaires out of the 71 included on D0). Because radiotherapy may impact specific functions (e.g., mucositis may impair swallowing) after a 15-day delay, one patient who filled in his first CHI less than one month after the end of treatment was excluded from this analysis. On average, patients completed the second questionnaire nine days after the first. The correlation coefficient for the "Changes in physical appearance" dimension was $r=0.67$ $(p<0.001)$ compared to $r=0.78(p<0.001)$ for the "Limitation of neck and/or shoulder movements" dimension.

\subsection{Link between the Carcinologic Handicap Index results and prioritisation of} patients' needs (secondary objective)

Calculation of the intraclass correlation coefficient (ICC) per subject between the two rankings (ranking of needs and ranking based on $\mathrm{CHI}$ ) revealed relatively high ICCs with a peak ranging from 0.6 to 0.8 . The confidence interval of this ICC does not include a value of 1 in 54\% of cases (38/70). There is a significant or suggestive difference between the two rankings.

Considering the top three dimensions in both rankings, there is a connection between 2 symptoms for $56 \%$ of patients.

The AUC (area under the curve) with its $95 \%$ confidence interval and the chosen cut-off value (with sensitivity, specificity and corresponding items ranked correctly) were calculated for each dimension (Table 3). The threshold parameters were not calculated for an AUC confidence interval of 0.50 , thus demonstrating poor screening performance in the dimension.

\section{Discussion}

\subsection{Validity and reliability of the upgraded CHI version}


The two new domains added to the CHI possess good psychometric qualities and can be seamlessly integrated into the overall questionnaire, as shown by the convergent and Two points should be noted regarding the analysis of the two new dimensions. Patients' age and sex may influence their answers to items in the "Changes in physical appearance" dimension. Treatments are often mutilating and contribute to degrading the physical appearance of the face. Indeed, younger and professionally active patients may for example have a different perception of the impact of the aesthetic changes following treatment of their cancer.

Regarding the "Limitations of neck and/or shoulder movement" dimension, the first question can be considered ambiguous. Indeed, patients are asked about the change in sensitivity in their neck, shoulders and/or arms. However, it does not directly question the notion of pain in the shoulders, which is frequent in the case of neck dissections [17]. However, perceived pain in these anatomical structures will still be reported by patients in the "Pain" dimension.

The scores obtained for the inter-domain correlation of the entire questionnaire (see Appendix 2) are relatively low, which confirms that the domains focus on different areas. This last conclusion should be weighted on the basis of three observations.

The strong correlation between the Swallowing and Feeding dimensions is probably linked to the first two questions, $\mathrm{N} 1$ and $\mathrm{N} 2$, which concentrate on the symptoms directly related to swallowing difficulties. The Feeding domain can therefore be partly used to assess the impact of swallowing problems on the patient's nutritional status.

The "Psychosocial" dimension is a cross-sectional domain, rapidly impacted by the onset of impaired function. The strongest correlations in this dimension ( $r>0.60)$ are apparent in "Phonation" and, above all, in the CHI "Changes in physical appearance" dimension. Psychosocial aspects are therefore known to primarily impact the dimensions involved in interpersonal communication, through speech or physical appearance. Although the other correlations are weaker $(\mathrm{r}<0.50)$, they are nevertheless still informative because they allow the therapist to gain an insight into how the individual is feeling overall as a result of the treatment sequelae. However, this questions the impact of a high score in this domain on interpreting the questionnaire. Given the strong correlations observed $(r>0.60)$, the "Psychosocial" dimension appears to influence how patients score the "Phonation" and "Changes in physical appearance" dimensions. An additional analysis was carried out to assess this point by weighting the scores of both these dimensions with a strong "Psychosocial" correlation using simple robust regression analysis (with the target dimension score expressed as the dependent variable and the Psychosocial score as the explanatory variable). Each time the psychosocial score increases by one point, the "Changes in physical appearance" score increases by 0.60 point $(\mathrm{p}<0.001)$ and the "Phonation" score by 0.67 point $(\mathrm{p}<0.001)$. The scores allocated to other dimensions with higher cohorts could also be analysed in this way to establish whether dimension scores should be qualified according to the "Psychosocial" score to facilitate a more precise interpretation of CHI results.

In terms of the other more weakly correlated dimensions, is the psychosocial difficulty due to other deficiencies listed in the CHI? This dimension, which refers to the prioritisation of human needs according to Marslow [18], is probably a reflection of the physiological needs at stake and their impact on other human needs. This mainly concerns the need for security, which also changes because an individual has been diagnosed with cancer, and the impact on appearance and self-esteem. A marked psychosocial impact through these strong correlations with certain dimensions may therefore lead to a change in scoring, regardless of the extent of the deficiency. 
The other psychometric analyses of the validity and reliability of the upgraded version of the $\mathrm{CHI}$ confirm the relevance of the questionnaire for an ENT cancer cohort with particular questionnaire scores and the way in which patients rate their difficulty in VAS terms, it can be concluded that the two new dimensions clearly portray the difficulty perceived by patients. The CHI was built to address the needs of patients in order to tailor the care strategy. It is deliberately generic, so that it can be administered to all patients with a deficit or complaint regarding upper aerodigestive tract function, whether or not the initial tumor location is in the oro-pharyngeal area. While patients' complaints and needs may differ depending on the tumor location, the treatment method, the age or the sex, the construction of this tool allows to take these factors into account. The sub-scores corresponding to each dimension will highlight the deficits that predominantly affect each patient, according to these factors. Moreover, the validation of the modular aspect of this upgraded version of the $\mathrm{CHI}$ allows to adapt the choice of dimensions presented to the patient to a specific complaint depending for example on the initial tumor location. However, the calculation of threshold scores requires further clarification. It is indeed likely that these scores differ according to tumour location with specific functional consequences in the case of oral or breast cancer with an ENT complaint for example. This study should therefore be continued to increase the number of patients treated specifically for UAT and calculating specific threshold scores for this population. However, there is no significant difference between the control and patient populations for certain dimensions such as Pain and Vision. These results are similar to those obtained during the initial CHI validation [12]. Compared to the initial publication, this study shows the "disappearance" of insignificance for the hearing domain, which is linked to the fact that the general population was represented by twice the size of the original sample used in the first validation (36 individuals compared to 18). Apart from the statistical problem of sample size and potential lack of power, these dimensions also focus on current problems, the frequency of which increases with age and which are therefore easily identifiable in our study populations.

The results of internal consistency, investigated using Cronbach's alpha coefficients, demonstrate good homogeneity for both domains.

\subsection{Determination of patient needs in adopting a therapeutic strategy}

The upgraded CHI version prioritises patient needs, not for the entire questionnaire but for the initial symptoms with the highest scores.

Firstly, any decisions regarding management needs cannot be based on the top score alone since it is not necessarily indicative of the most debilitating symptom as far as the patient is concerned. This means that the practitioner should not focus on the cardinal symptom and highlight this as the cause of all the patient's problems. On the other hand, it is not necessarily practical to take into consideration all symptoms with a score above zero because the domains with the lowest CHI scores are often barely debilitating for patients. Indeed they often rank at the bottom in terms of prioritised needs, or are not even ranked at all.

Hence, the trend in current clinical practice to consider the associated functional impact is perfectly in line with the WHO's International Classification of Functioning. The CHI directly targets activity limitations and participation restrictions in order to tailor care for each patient. Moreover, the CHI should be used early on and at regular intervals during the patient's follow-up. The expressed needs may change according to the recovery progress and the chronic nature of the disease. The $\mathrm{CHI}$ is a suitable tool to best adapt the therapeutic strategy of each patient over time. 
Conversely, this study shows that it is relevant in clinical practice to take into consideration three to five symptoms with the highest $\mathrm{CHI}$ scores. The questionnaire prioritises patient needs, not for the entire questionnaire per se but for those symptoms with the highest scores. The latter coincide with the patient's feelings and expectations. In clinical practice, good psychometric results were recorded when screening priority areas for the patient, primarily in the following domains: "Swallowing", "Phonation", "Hearing", "Olfaction-Gustation" and "Feeding" (area under the curve $>0.74$ and ranking performance $>75 \%$ ). The screening performances for the "Pain", "Respiration" and "Limitation of neck and/or shoulder movements" dimensions are still acceptable, albeit to less of an extent. The "Psychosocial impact" and "Changes in physical appearance" domains in turn, are not significant in terms of screening performance. Thus, although the threshold scores seem to be feasible for eight out of the eleven dimensions, use of the CHI should still be supported by patient discussion to establish the impact of the problem and the importance attached to it in terms of "Psychosocial impact", "Changes in physical appearance" and "Vision".

\subsection{Perspectives}

Although the $\mathrm{CHI}$ was built to assess the dimensions by asking patients about their general complaints after cancer treatment, environmental, socio-educational and cultural factors may modify the perception of deficits. Therefore, the CHI might need to be revalidated before being used in populations from different socio-cultural backgrounds. The problem encountered by tube-fed patients is another important item for consideration in subsequent developments. Indeed, the wording of the "Swallowing" and "Feeding" questions does not allow tube-fed patients to 'voice' their problems. Given the strong correlation that generally exists between these two areas, it would seem useful to establish whether the rules governing use or interpretation could improve usage or whether both these dimensions should be reconstructed.

Furthermore, the psychosocial dimension is often scored but it does not always appear to be a priority for the patient (particularly for tube-fed patients, which corroborates our earlier analysis in this area). It is assumed that, faced with "physical" symptoms such as swallowing difficulties, speech problems or even pain, it is more difficult for patients to assess any feelings of isolation or depression. Moreover, this is once again a cross-sectional domain and questions are answered in the affirmative as soon as treatment sequelae impact daily life, albeit not to any considerable extent.

It is therefore entirely appropriate to consider a modular CHI format where patients are questioned on only one or more specific dimensions. As the perceived needs may differ depending on the age, professional activity, sex, tumor location, treatment and functional recovery, an evaluation of the relevance of some dimensions according to these parameters and the patient's profile could be carried out in a future study.

The use of an overall cumulative score does not seem relevant given the heterogeneity of the dimensions tested and the fact that patients' feelings could be linked to the tumour site, for instance, or to the psychosocial dynamics in which individual patients find themselves.

\section{Conclusion}

Thanks to its good psychometric properties and appropriate clinical use, the CHI allows practitioners to steer the consultation directly to these symptoms or even to refer the patient to another professional, if required. CHI performance should ideally be compared to a 
395 questionnaire such as the Patient Concerns Inventory to check its validity in terms of the 396 merits of CHI as a tool for steering patient/therapist consultations.

397 Finally, we suggest that the upgraded version of the CHI be renamed to align this tool more 398 accurately with the underlying objectives: "Head and Neck Carcinologic Handicap Index 399 (HN-CHI)". 


\section{Bibliographical references}

[1] Mlynarek A., Rieger J., Harris J., et al. Methods of functional outcomes assessment following treatment of oral and oropharyngeal cancer: review of the literature. $J$ Otolaryngol Head Neck Surg. 2008; 37(1) : 2-10.

[2] Reich M. Cancer and Body Image : Identity, Representation. Inf Psych. 2009; 85(3): 247254. https://doi.org/10.3917/inpsy.8503.0247

[3] Borggreven P. A., Verdonck-De Leeuw I. M., Muller M. J., et al. Quality of life and functional status in patients with cancer of the oral cavity and oropharynx: Pretreatment values of a prospective study. Eur Arch Otorhinolaryngol. 2007; 264(6) : 651-657. https://doi.org/10.1007/s00405-007-0249-5

[4] DeNittis A. S., Machtay M., Rosenthal D. I., et al. Advanced oropharyngeal carcinoma treated with surgery and radiotherapy: Oncologic outcome and functional assessment. Am J Otolaryngol. 2001; 22(5): 329-335. https://doi.org/10.1053/ajot.2001.26492

[5] Stelzle F., Knipfer, C. Schuster M., et al. Factors influencing relative speech intelligibility in patients with oral squamous cell carcinoma: A prospective study using automatic, computer-based speech analysis. Int J Oral Maxillofac Surg. 2013 ; 42(11) : 1377-1384. https://doi.org/10.1016/j.ijom.2013.05.021

[6] Murphy B. A., Ridner S., Wells N., Dietrich M. Quality of life research in head and neck cancer: A review of the current state of the science. Crit Rev Oncol Hematol. 2007; 62(3): 251-267. https://doi.org/10.1016/j.critrevonc.2006.07.005

[7] Thomas L., Jones T. M., Tandon S., Carding P., Lowe D., Rogers S. Speech and voice outcomes in oropharyngeal cancer and evaluation of the University of Washington Quality of Life speech domain. Clin Otolaryngol. 2009; 34(1): 34-42. https://doi.org/10.1111/j.1749-4486.2008.01830.x

[8] Bjordal B. K., Hammerlid E., Ahlner-elmqvist M., et al. Quality of Life in Head and Neck Cancer Patients : Validation of the European Organization for Research and Treatment of Cancer Quality of Life Questionnaire - H\&N35. J Clin Oncol. 2016 ; 17(3): 10081019.

[9] Rinkel R. N., Leeuw I. M. V., Van-Reij E. J., Aaronson N. K., Leemans R. Speech Handicap Index in patients with oral and pharyngeal cancer: better understanding of patients' complaints. Head Neck. 2008; 30: 868-874. https://doi.org/10.1002/HED

[10] Balaguer M., Farinas J., Fichaux-Bourin P., Puech M., Pinquier J., Woisard, V. Validation of the French Versions of the Speech Handicap Index and the Phonation Handicap Index in Patients Treated for Cancer of the Oral Cavity or Oropharynx. Folia Phoniatr Logop. 2019 ; 15: 1-14. https://doi.org/10.1159/000503448

[11] Rogers S. N., El-Sheikha J., \& Lowe D. The development of a Patients Concerns Inventory (PCI) to help reveal patients concerns in the head and neck clinic. Oral Oncol. 2009; 45(7): 555-561. https://doi.org/10.1016/j.oraloncology.2008.09.004

[12] Rogers S. N., Lowe D., Lowies C., et al. Improving quality of life through the routine use of the patient concerns inventory for head and neck cancer patients: A cluster preference randomized controlled trial. BMC Cancer. 2018; 18(1), 1-10. https://doi.org/10.1186/s12885-018-4355-0

[13] Balaguer M., Percodani J., Woisard V. The Carcinologic Handicap Index (CHI): A disability self-assessment questionnaire for head and neck cancer patients. Eur Ann Otorhinolaryngol Head Neck Dis. 2017; 134(6): 399-403. https://doi.org/10.1016/j.anorl.2017.06.010

[14] Mokkink L. B., Terwee C. B., Patrick D. L., et al. The COSMIN study reached international consensus on taxonomy, terminology, and definitions of measurement properties for health-related patient-reported outcomes. J Clin Epidemiol. 2010 ;63(7), 
737-745. https://doi.org/10.1016/j.jclinepi.2010.02.006

[15] Ioannidis, J. P. A. The proposal to lower P value thresholds to .005. 2018; 319(14), 1429-1430. https://doi.org/10.1001/jama.2018.1536

[16] Laccourreye, O., Lisan, Q., Bonfils, P., et al. Use of P-values and the terms "significant", "non-significant" and "suggestive" in Abstracts in the European Annals of Otorhinolaryngology, Head \& Neck Diseases. Eur Ann of Otorhinolaryngol Head Neck Dis. 2019; 136(6), 469-473. https://doi.org/10.1016/j.anorl.2019.10.008

[17] Manning, M., Stell, P. M. The shoulder after radical neck dissection. Clinical Otolaryngology. 14 ; 5: 381-384. doi:10.1111/j.1365-2273.1989.tb00389.x

[18] Maslow A. H. A theory of human motivation. Psychological Review. 1943 ; 50(4), 370396. 


\begin{tabular}{|c|c|c|c|c|c|}
\hline & \multirow[b]{2}{*}{ Variable } & \multicolumn{2}{|c|}{ Patients } & \multicolumn{2}{|c|}{ Controls } \\
\hline & & Frequency & Percentage & Frequency & Percentage \\
\hline \multirow[t]{2}{*}{ Gender } & Male & 46 & $64.79 \%$ & 17 & \\
\hline & Female & 25 & $35.12 \%$ & 19 & \\
\hline \multirow[t]{2}{*}{ Age } & In years: mean (sd) & & 64.54 (10.15) & & $59.53(10.23)$ \\
\hline & $\begin{array}{r}\text { In years: median } \\
{[\mathrm{P} 25 ; \mathrm{P} 75]} \\
\end{array}$ & & $65[60 ; 72]$ & & $58[55 ; 60.5]$ \\
\hline \multirow[t]{5}{*}{ Tumour site } & Oral cavity & 14 & $19.72 \%$ & & \\
\hline & Larynx & 15 & $21.13 \%$ & & \\
\hline & Pharynx & 21 & $29.58 \%$ & & \\
\hline & Other ENT sites 1 & 5 & $7.04 \%$ & & \\
\hline & $\begin{array}{r}\text { Other site (non- } \\
\text { ENT) }{ }^{2}\end{array}$ & 16 & $22.54 \%$ & & \\
\hline \multirow[t]{3}{*}{ Surgery } & No & 20 & $28.17 \%$ & & \\
\hline & Yes & 48 & $67.61 \%$ & & \\
\hline & Not involved & 3 & $4.23 \%$ & & \\
\hline \multirow[t]{3}{*}{ Radiotherapy } & No & 12 & $16.90 \%$ & & \\
\hline & Yes & 56 & $78.87 \%$ & & \\
\hline & Not involved & 3 & $4.23 \%$ & & \\
\hline \multirow[t]{3}{*}{ Chemotherapy } & No & 33 & $46.48 \%$ & & \\
\hline & Yes & 35 & $49.30 \%$ & & \\
\hline & Not involved & 3 & $4.23 \%$ & & \\
\hline \multirow{3}{*}{$\begin{array}{c}\text { Other treatment } \\
\text { (clinical trial, } \\
\text { immunotherapy, IRA } \\
\text { therapy, laser, } \\
\text { hormone therapy) }\end{array}$} & No & 59 & $83.10 \%$ & & \\
\hline & Yes & 9 & $12.68 \%$ & & \\
\hline & Not involved & 3 & $4.23 \%$ & & \\
\hline $\begin{array}{l}\text { Length of time since } \\
\text { end of treatment }\end{array}$ & $\begin{array}{r}\text { In months: median } \\
\text { [P25; P75] }\end{array}$ & $21[6 ; 84]$ & & & \\
\hline
\end{tabular}

Table 1: description of subjects enrolled in the study

${ }^{1}$ Cervical adenopathy with no primary, thyroid

2 Breast, acute leukaemia, CUP (Carcinoma with Unknown Primary tumour) syndrome, retro-orbital melanoma, prostate, lungs, ovary, paraganglioma, brain stem 


\begin{tabular}{|l|l|l|l|l|l|l|}
\hline \multirow{2}{*}{} & \multicolumn{5}{|c|}{$\begin{array}{l}\text { Limitation of neck and/or shoulder } \\
\text { movements }\end{array}$} & \multicolumn{2}{c|}{$\begin{array}{c}\text { Changes in physical appearance } \\
\text { (Lowest intra-domain correlation: r=0.374) }\end{array}$} \\
\hline Dimension & $\begin{array}{l}\text { Highest } \\
\text { coefficient }\end{array}$ & $\begin{array}{l}\text { Lowest } \\
\text { coefficient }\end{array}$ & $\begin{array}{l}\text { Number of } \\
\text { inter-domain } \\
\text { correlation } \\
\text { r>0.351 }\end{array}$ & $\begin{array}{l}\text { Highest } \\
\text { coefficient }\end{array}$ & $\begin{array}{l}\text { Lowest } \\
\text { coefficient }\end{array}$ & $\begin{array}{l}\text { Number of } \\
\text { inter-domain } \\
\text { correlation } \\
\text { r }>0.374\end{array}$ \\
\hline Pain & 0.24 & 0.04 & 0 & 0.37 & 0.03 & 0 \\
\hline Swallowing & 0.40 & 0.01 & 1 & 0.32 & 0.11 & 0 \\
\hline Feeding & 0.29 & 0.00 & 0 & 0.42 & 0.05 & 2 \\
\hline Respiration & 0.36 & 0.04 & 1 & 0.42 & 0.08 & 1 \\
\hline Phonation & 0.29 & 0.03 & 0 & 0.50 & 0.12 & 3 \\
\hline Hearing & 0.21 & 0.03 & 0 & 0.14 & 0.00 & 0 \\
\hline Vision & 0.22 & 0.01 & 0 & 0.42 & 0.06 & 1 \\
\hline Olfaction-Gustation & 0.28 & 0.02 & 0 & 0.44 & 0.05 & 1 \\
\hline $\begin{array}{l}\text { Changes in physical } \\
\text { appearance }\end{array}$ & 0.30 & 0.05 & 0 & $/$ & $/$ & $/$ \\
\hline $\begin{array}{l}\text { Limitation of neck and/or } \\
\text { shoulder movements }\end{array}$ & $/$ & $/$ & $/$ & 0.30 & 0.05 & 0 \\
\hline Psychosocial impact & 0.41 & 0.07 & 2 & 0.80 & 0.38 & 16 \\
\hline
\end{tabular}

Table 2 Detailed absolute values of inter-domain correlation scores for items in the following dimensions, namely, "Limitation of neck and/or shoulder movements" and "Changes in physical appearance" 


\begin{tabular}{|c|c|c|c|c|c|c|}
\hline \multirow[t]{2}{*}{ Domain } & \multirow{2}{*}{$\begin{array}{l}\text { AUC (Area } \\
\text { Under the } \\
\text { Curve) }\end{array}$} & \multirow{2}{*}{$\begin{array}{c}95 \% \text { CI of the } \\
\text { AUC }\end{array}$} & \multicolumn{4}{|c|}{ Determination of the cut-off value } \\
\hline & & & $\begin{array}{l}\text { Cut-off } \\
\text { value } \\
\text { chosen } \\
\text { (dimension } \\
\text { score) }\end{array}$ & Sensitivity & Specificity & $\begin{array}{l}\text { Ranked } \\
\text { correctly }\end{array}$ \\
\hline Pain & 0.73 & {$[0.58 ; 0.87]$} & 7 & $64.7 \%$ & $73.6 \%$ & $71.4 \%$ \\
\hline Swallowing & 0.84 & {$[0.73 ; 0.94]$} & 4 & $93.6 \%$ & $56.5 \%$ & $81.4 \%$ \\
\hline Feeding & 0.78 & {$[0.67 ; 0.89]$} & 7 & $80.8 \%$ & 72.7\% & $75.7 \%$ \\
\hline Respiration & 0.74 & {$[0.54 ; 0.94]$} & 4 & $87.5 \%$ & $50.0 \%$ & $54.3 \%$ \\
\hline Phonation & 0.85 & {$[0.74 ; 0.95]$} & 6 & $90.9 \%$ & $65.4 \%$ & $81.4 \%$ \\
\hline Hearing & 0.88 & {$[0.79 ; 0.96]$} & 8 & $83.3 \%$ & $81.0 \%$ & $81.4 \%$ \\
\hline Vision & 0.55 & {$[0.35 ; 0.75]$} & $\mathrm{ND}$ & ND & ND & ND \\
\hline Olfaction-Gustation & 0.86 & {$[0.69 ; 1.00]$} & 7 & $\mathbf{7 7 . 8 \%}$ & $85.3 \%$ & $84.3 \%$ \\
\hline $\begin{array}{l}\text { Changes in physical } \\
\text { appearance }\end{array}$ & 0.68 & {$[0.49 ; 0.88]$} & ND & ND & ND & ND \\
\hline $\begin{array}{l}\text { Limitation of neck } \\
\text { and/or shoulder } \\
\text { movements }\end{array}$ & 0.70 & {$[0.57 ; 0.83]$} & 7 & $70.8 \%$ & $52.2 \%$ & $58.6 \%$ \\
\hline $\begin{array}{l}\text { Psychosocial } \\
\text { impact }\end{array}$ & 0.48 & {$[0.21 ; 0.75]$} & ND & ND & ND & ND \\
\hline
\end{tabular}

Table 3 Psychometric performances of various CHI dimensions in screening priority management areas for patients (ND: Not Determined) 
Please tick the box that best describes your current situation where:

$\mathrm{N}=$ never; $\mathrm{AN}=$ almost never; $\mathrm{S}=$ sometimes; $\mathrm{AA}=$ almost always; $\mathrm{A}=$ always

\begin{tabular}{|c|c|c|c|c|c|c|}
\hline & & $\mathbf{N}$ & AN & $\mathbf{S}$ & $\mathbf{A A}$ & $\mathbf{A}$ \\
\hline PA1 & Do you take medication for pain? & & & & & \\
\hline PA2 & Do you wake up at night because of pain? & & & & & \\
\hline PA3 & Are you hindered in your activities by pain? & & & & & \\
\hline PA4 & Do you have pain attacks? & & & & & \\
\hline SW1 & Do you have discomfort swallowing? & & & & & \\
\hline SW2 & Do you ever choke whilst eating or drinking? & & & & & \\
\hline SW3 & Do you have liquid or solid reflux after eating? & & & & & \\
\hline SW4 & Do you have problems chewing? & & & & & \\
\hline FE1 & Do you need to change the texture of food to swallow it? & & & & & \\
\hline FE2 & Do you take longer to eat due to difficulty in swallowing? & & & & & \\
\hline FE3 & Do you need to enrich your food? & & & & & \\
\hline FE4 & Have you lost weight? & & & & & \\
\hline RE1 & Do you have difficulty breathing at rest? & & & & & \\
\hline RE2 & Do your breathing problems restrict your physical activity? & & & & & \\
\hline RE3 & Do you feel congested? & & & & & \\
\hline RE4 & Do you need to be semi-seated to sleep? & & & & & \\
\hline PH1 & Do you have difficulty speaking? & & & & & \\
\hline $\mathrm{PH} 2$ & Do people have difficulty understanding you? & & & & & \\
\hline $\mathrm{PH} 3$ & Do you speak less with your family, friends, neighbours? & & & & & \\
\hline PH4 & Do you have difficulty articulating? & & & & & \\
\hline HE1 & Do you ask people to repeat themselves when talking to you? & & & & & \\
\hline HE2 & Do you have problems following conversations in a noisy environment? & & & & & \\
\hline HE3 & Do you have problems understanding over the phone? & & & & & \\
\hline HE4 & Do you have noises in your ears? & & & & & \\
\hline VI1 & Do you have difficulty seeing in the shade? & & & & & \\
\hline VI2 & Do you see less well at a distance or close up? & & & & & \\
\hline VI3 & Are you bothered by dazzle or floaters in your eyes? & & & & & \\
\hline VI4 & Has your visual field shrunk? & & & & & \\
\hline OG1 & Do you have difficulty smelling? & & & & & \\
\hline OG2 & Are you afraid of having an accident because of your difficulty smelling? & & & & & \\
\hline OG3 & Do you have difficulty tasting food? & & & & & \\
\hline OG4 & Do you feel frustrated by your difficulty smelling and tasting? & & & & & \\
\hline CP1 & Are you bothered by changes in your physical appearance? & & & & & \\
\hline $\mathrm{CP} 2$ & Are you bothered by the way other people look at you since your illness? & & & & & \\
\hline $\mathrm{CP} 3$ & Is your social life limited by changes in your physical appearance? & & & & & \\
\hline $\mathrm{CP} 4$ & Has your personal life changed because of your illness? & & & & & \\
\hline LI1 & Have you noticed any change in sensitivity in your neck, shoulders and/or arms? & & & & & \\
\hline LI2 & Do you have difficulty turning your head? & & & & & \\
\hline LI3 & Do you have weight-bearing difficulties? & & & & & \\
\hline LI4 & Are you limited in raising your arms? & & & & & \\
\hline PS1 & Is your personal and social life limited by problems related to your illness? & & & & & \\
\hline PS2 & Has your illness affected your relationship with other people? & & & & & \\
\hline PS3 & Are you bothered by problems related to your illness? & & & & & \\
\hline PS4 & Do you feel handicapped by your illness? & & & & & \\
\hline
\end{tabular}




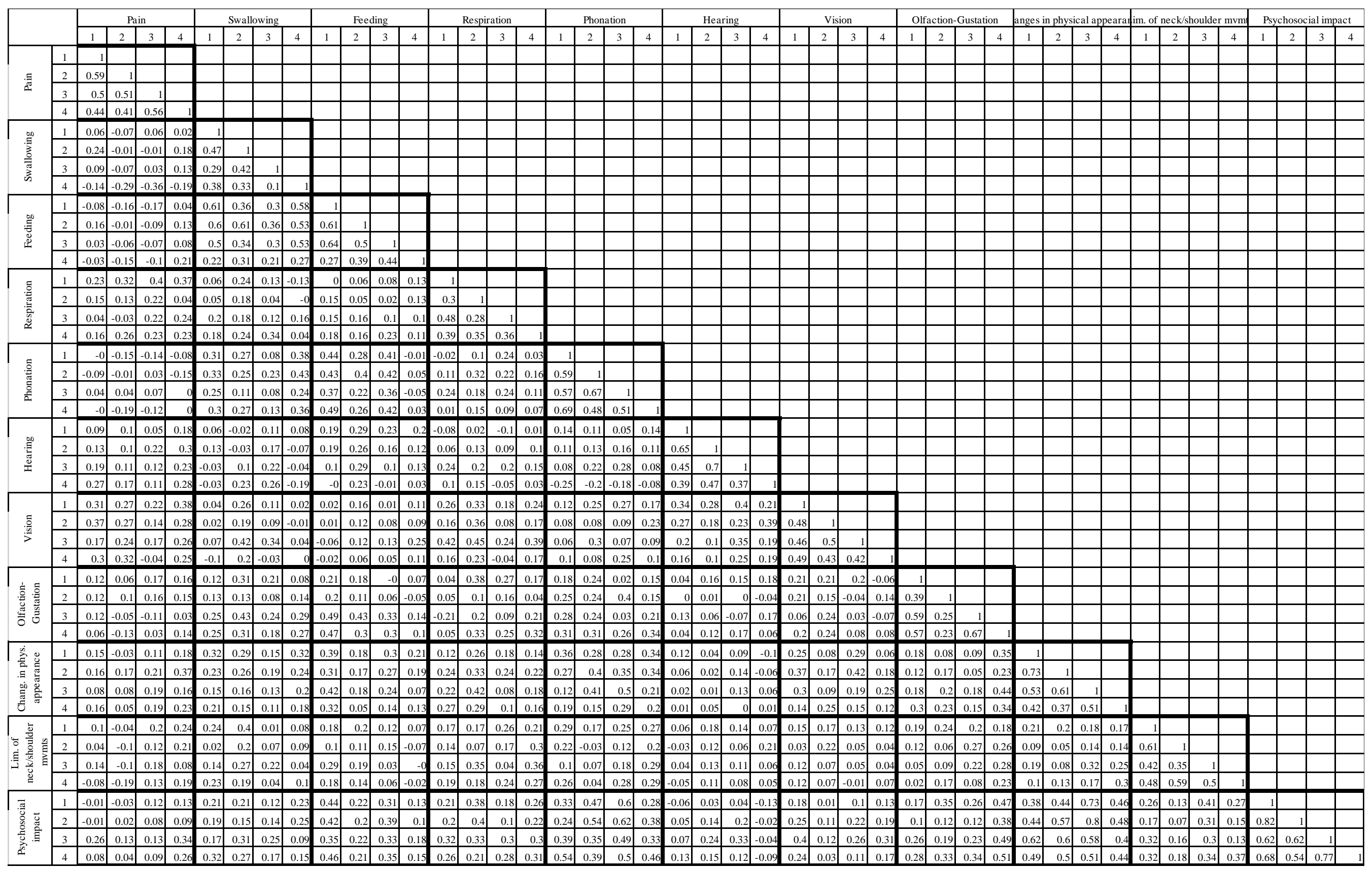

